\title{
EFFECT OF RIB ROOT SHAPE ON THE TENSILE STRENGTH OF PRESS AND INJECTION HYBRID MOLDED PRODUCTS MADE WITH CF/PA6 RANDOM PREPREG SHEET
}

\author{
KAZUTO TANAKA ${ }^{1}$, MASAOMI KAETSU ${ }^{1}$, TSUTAO KATAYAMA ${ }^{1} \&$ TAKESHI ISHIKAWA $^{2}$ \\ ${ }^{1}$ Department of Biomedical Engineering, Doshisha University, Japan \\ ${ }^{2}$ Mitsubishi Chemical Co., Japan
}

\begin{abstract}
The use of carbon fiber reinforced thermoplastic (CFRTP) contributes to weight reduction of automobiles and offers better gasoline mileage. Recently, to mold CFRTP with high-strength and highstiffness for complex shaped products with ribs and bosses, the hybrid molding system has been developed by combining press molding and injection molding. Although rib root shape is the important factor in the press and injection hybrid molding, the effect of rib root shape on the rib root strength has not been clarified yet. In this study, carbon fiber reinforced polyamide 6 composites were molded by press and injection hybrid molding. Rib root tensile test of T-shaped specimen cut out from the hybrid molded products and in-plain tensile test of the outer shell laminate were conducted to clarify the effect of rib root shape (radius of curvature $\mathrm{R}=0$ and 2.5). For rib root tensile test of $\mathrm{T}$-shaped specimens, higher strength was obtained in the molded products with $\mathrm{R}=0$ than those with $\mathrm{R}=2.5$. On the other hand, in the case of in-plain tensile strength of outer shell laminate, higher tensile strength was obtained in the molded products with $\mathrm{R}=2.5$ than those with $\mathrm{R}=0$.

Keywords: CFRTP, press and injection hybrid molding, rib root shape, rib root tensile test, in-plain tensile test, random prepreg sheet.
\end{abstract}

\section{INTRODUCTION}

The use of CFRTP contributes to weight reduction of automobiles and offers better gasoline mileage [1], [2]. In the automobile industry, reducing vehicle weight to reduce energy consumption and carbon dioxide emissions is one of the major issues [3].

Recently, to mold CFRTP with high-strength and high-stiffness for complex shaped products with ribs and bosses, the hybrid molding system has been developed by combining press molding and injection molding [4]. In our previous study, it has been reported that fracture occurs at the interface between laminates and ribs for the press and injection hybrid molding [5]. To improve the interfacial strength, supplying method of discontinuous fibers to the interface between laminates and ribs was proposed, and the oriented discontinuous fiber in the rib to vertical direction to the interface contributed the improvement of the interfacial strength [6], [7]. Although rib root shape or radius of curvature is the important factor in the press and injection hybrid molding, the effect of rib root shape on the mechanical property has not been clarified yet.

In this study, carbon fiber reinforced polyamide 6 composites were molded by press and injection hybrid molding by using polyamide 6 random prepreg sheet for outer shell laminate material. Rib root tensile tests of T-shaped specimen and in-plain tensile strength of the outer shell laminate specimens cut out from the molded products were conducted to clarify the effect of rib root shape on their mechanical properties. 


\section{MATERIALS AND EXPERIMENTAL PROCEDURE}

\subsection{Materials}

Carbon fiber polyamide 6 random prepreg sheet [8] (Product by Mitsubishi Chemical Co., Japan, $\mathrm{V}_{\mathrm{f}}=35 \%, 0.5 \mathrm{~mm}$ in thickness) was used for outer shell laminates of hybrid molding. The polyamide 6 (PA6, 1015B, Ube Industries Ltd., Japan) pellets were used for the materials of injection molding. The press and injection hybrid molding machine (Sato Machinery Works Co. Ltd., Japan) was used for manufacturing the laminated CF/PA6 random prepreg sheet and the hybrid CFRTP composites. The CF/PA6 random prepreg sheet laminate $(3 \mathrm{~mm}$ in thickness) was molded with a plate mold at $280^{\circ} \mathrm{C}$ and held at $2.0 \mathrm{MPa}$ pressure for 180 seconds.

\subsection{Press and injection hybrid molding}

A schematic drawing of the press and injection hybrid molding system is shown in Fig. 1. Table 1 shows the molding condition for the press and injection hybrid molding. The laminated $\mathrm{CF} / \mathrm{PA} 6$ random prepreg sheet were heated and melted in advance using the heater at $280^{\circ} \mathrm{C}$ for 360 seconds. After the laminated CF/PA6 random prepreg sheet was placed inside the mold, the laminate was press-molded at press pressures of $8 \mathrm{MPa}$. After that, PA6 pellet was injected into the space for the ribs and press pressure was set to $16 \mathrm{MPa}$. The molded product manufactured by the press and injection hybrid molding is shown in Fig. 2. This process can mold CFRTP with high-strength and high-stiffness for complex shaped products with ribs.

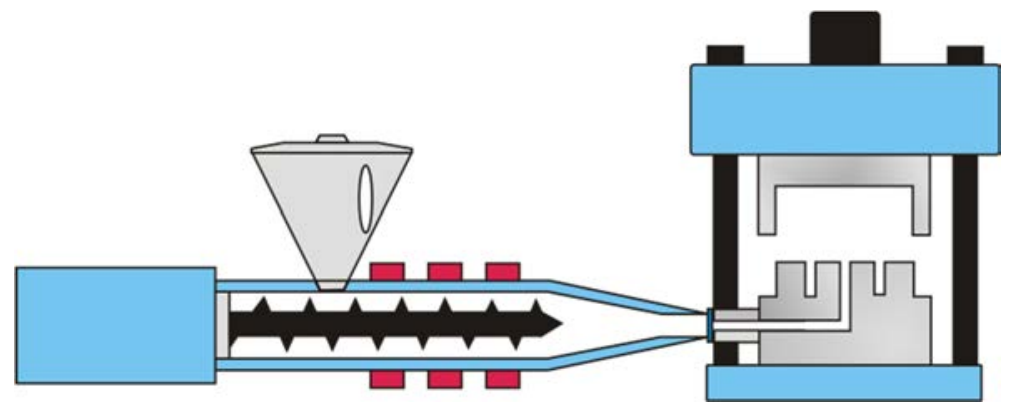

(a)
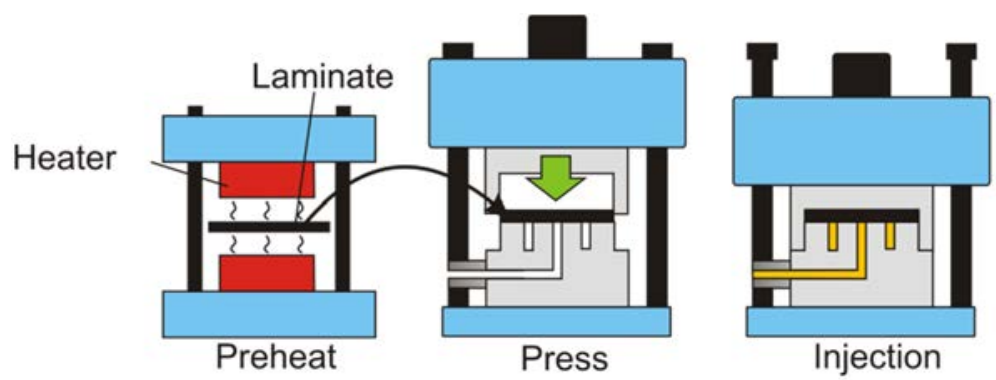

(b)

Figure 1: Schematic drawing of the press and injection hybrid molding process. (a) Hybrid molding system; and (b) Preheat and molding. 
Table 1: Molding conditions of the press and injection hybrid molding.

\begin{tabular}{|c|c|c|c|}
\hline \multicolumn{2}{|c|}{ Preheat } & \multirow{2}{*}{$\begin{array}{c}\text { First } \\
\text { pressure } \\
(\mathrm{MPa})\end{array}$} & $\begin{array}{c}\text { Second } \\
\text { pressure } \\
(\mathrm{MPa})\end{array}$ \\
\hline 280 & 360 & 8 & 16 \\
\hline
\end{tabular}

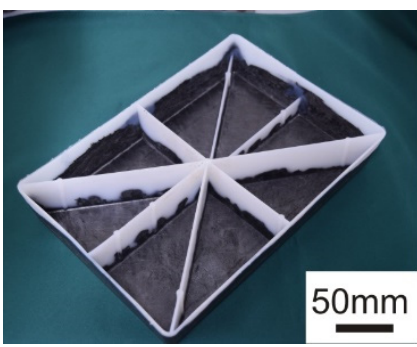

(a)

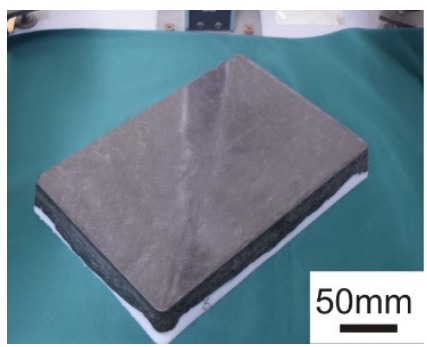

(b)

Figure 2: Molded product manufactured by the press and injection hybrid molding. (a) Upper side (ribs structure); and (b) Under side (laminates).

2.3 Rib root tensile test of T-shaped specimen and in-plain tensile test of the outer shell laminate

In order to evaluate the effect of rib root shape (radius of curvature $\mathrm{R}=0$ and 2.5 , shown in Fig. 3) on the rib root strength, penetrated height was measured and rib root tensile test of Tshaped specimen was conducted. Rib root T-shaped specimens were cut out from the hybrid molded products as shown in Fig. 4(a). Rib root tensile tests for T-shaped specimens were conducted with a cross speed of $0.017 \mathrm{~mm} / \mathrm{s}$ at room temperature with a universal testing machine (5566, Instron, USA). The dimensions of the specimens were $35 \mathrm{~mm}$ in length and $15 \mathrm{~mm}$ in width and aluminium tabs were bonded using epoxy adhesive.

In order to evaluate the effect of rib root shape (radius of curvature $\mathrm{R}=0$ and 2.5) on the tensile strength of the outer shell laminate, in-plain tensile tests of the outer shell laminate were conducted by the tensile testing machine (Auto graph, AG-100kNX, Shimadzu Co. Ltd., Japan) with a cross head speed of $0.017 \mathrm{~mm} / \mathrm{s}$ at the room temperature. A non-contact strain analysis system (ARAMIS ${ }^{\circledR}$, Gom GmbH, Germany) was used for the strain distribution of the specimen during the in-plain tensile test. This system uses the digital image correlation

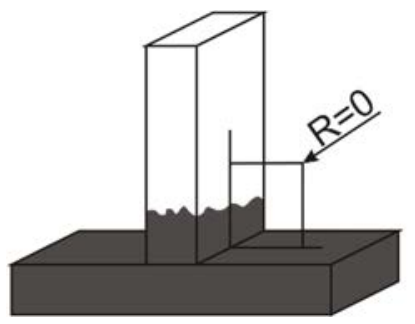

(a)

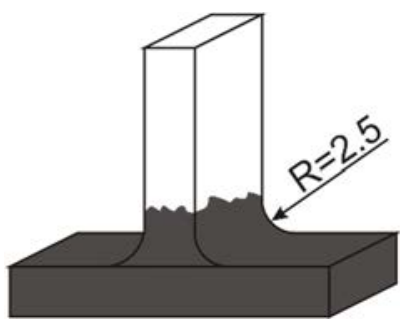

(b)

Figure 3: Schematic drawing of rib root shape. (a) $\mathrm{R}=0$; and (b) $\mathrm{R}=2.5$. 

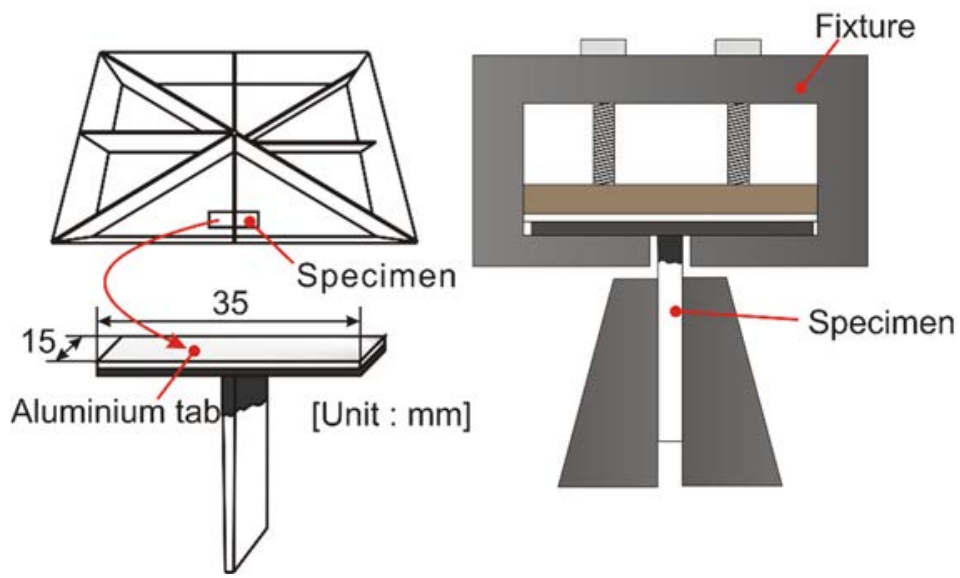

(a)

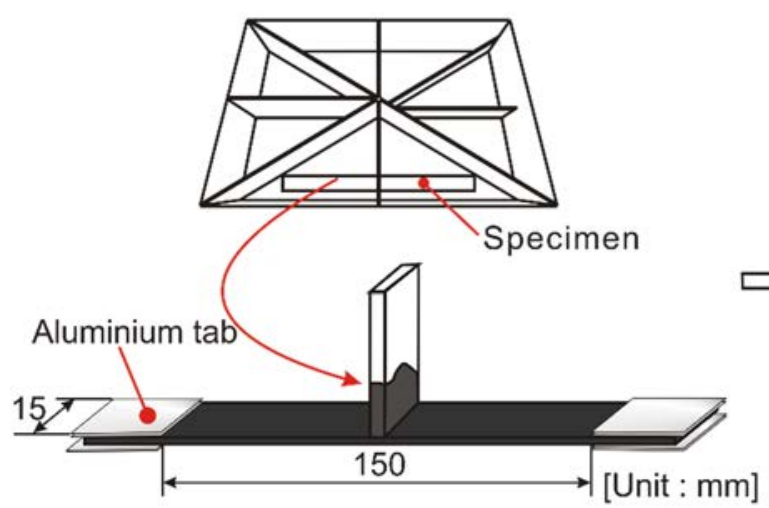

(b)

Figure 4: Schematic drawing of two types of tensile tests. (a) Rib root tensile test of Tshaped specimen; and (b) In-plain tensile test of outer shell laminate.

of random mark which were randomly added on the specimen surface by spray. The in-plain tensile test specimen was cut out from the hybrid molded products as shown in Fig. 4(b). The dimensions of the specimens were $150 \mathrm{~mm}$ in length and $15 \mathrm{~mm}$ in width and aluminium tabs were glued to the both ends of the specimen using epoxy adhesive.

\section{RESULTS AND DISCUSSIONS}

The cross-sectional views of $\mathrm{T}$-shaped specimen for $\mathrm{R}=0$ and $\mathrm{R}=2.5$ are shown in Fig. 5 . The carbon fiber of the CF/PA6 random prepreg sheet laminate penetrates into the rib structure. Fig. 6 shows the penetrated height of carbon fiber into the rib for T-shaped specimens. The penetrated height of specimens for $\mathrm{R}=2.5$ is higher than that for $\mathrm{R}=0$. Fig. 7 shows load-displacement curve and rib root tensile strength obtained by tensile tests of the rib root T-shaped specimens. The rib root tensile strength of T-shaped specimens for $\mathrm{R}=0$ is higher than that for $\mathrm{R}=2.5$ and the displacement of $\mathrm{T}$-shaped specimen for $\mathrm{R}=0$ is 


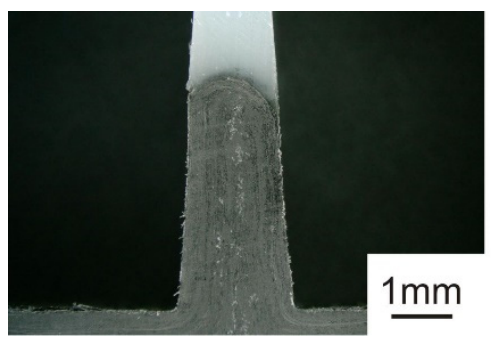

(a)

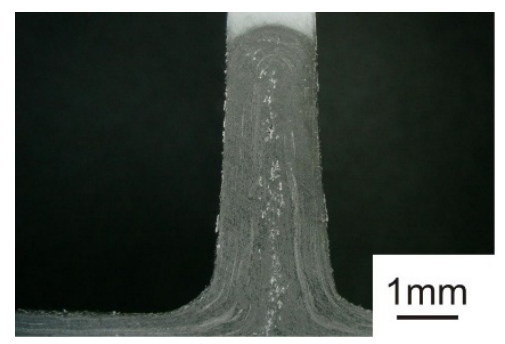

(b)

Figure 5: Cross-sectional views of $\mathrm{T}$-shaped specimen for (a) $\mathrm{R}=0$; and (b) $\mathrm{R}=2.5$.

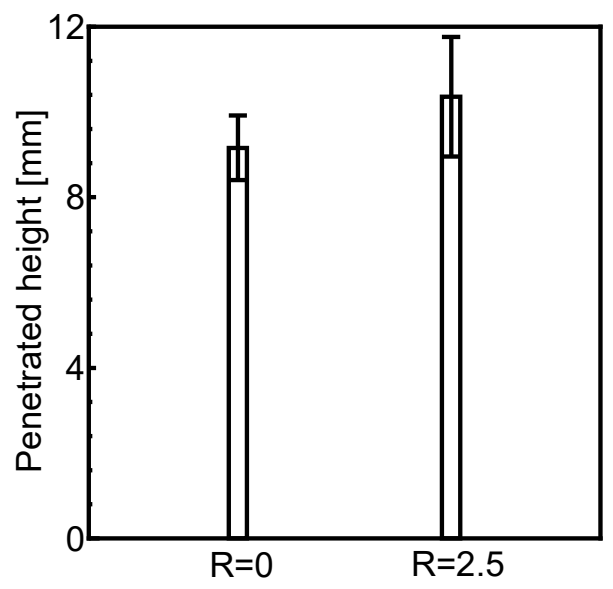

Figure 6: Penetrated height of carbon fiber into the rib for T-shaped specimens.

larger than that for $\mathrm{R}=2.5$. Although the stress concentration at the rib root corner at $\mathrm{R}=0$ is larger than that at $\mathrm{R}=2.5$ as shown in Fig. 3, the T-shaped specimen with the rib root shape for $\mathrm{R}=0$ has the higher rib root tensile strength. To obtain higher rib root tensile strength, the rib root shape of $\mathrm{T}$-shaped specimen for $\mathrm{R}=0$ is recommended for the press and injection hybrid molded products.

Fig. 8 shows the fracture surfaces of $\mathrm{T}$-shaped specimens for $\mathrm{R}=0$ and $\mathrm{R}=2.5$. While fracture occurred in the PA6 and at the interface between the laminates and injected materials of PA6 regardless of the rib root shapes, the surface area fractured in PA6 material for $\mathrm{R}=0$ is larger than that for $\mathrm{R}=2.5$. The tensile strength of PA6 is $64.0 \pm 0.7 \mathrm{MPa}$. The obtained rib root tensile strength as shown in Fig. 7(b) is lower than the tensile strength of PA6. We reported that the oriented discontinuous fiber in the rib to vertical direction to the interface contributed the improvement of the interfacial strength [6], [7]. Fiber orientation at the interface between the laminates and injected materials is considered to be the reason for the difference of rib root tensile strength and fracture surface. It is important to observe the fiber orientation at the interface using X-ray CT. This will be the future work.

Fig. 9 shows the in-plain tensile strength of outer shell laminate for $\mathrm{R}=0$ and $\mathrm{R}=2.5$. The in-plain tensile strength of outer shell laminate for $\mathrm{R}=2.5$ is higher than that for $\mathrm{R}=0$. Fig. 10 shows strain distribution of outer shell laminate for the in-plain tensile test at $\varepsilon=0.3 \%$. 


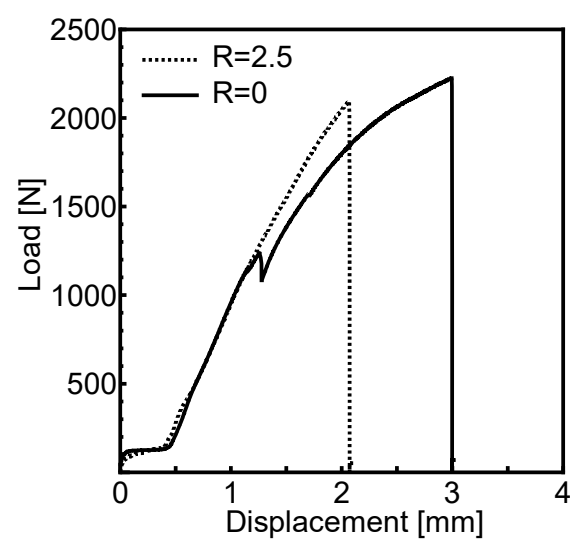

(a)

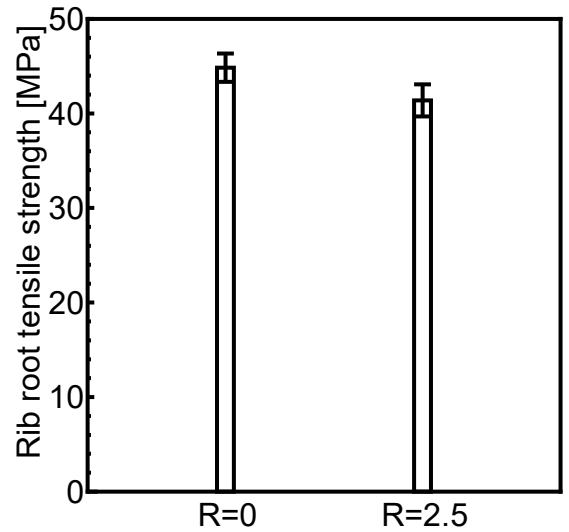

(b)

Figure 7: Result of $\mathrm{T}$-shaped tensile test for $\mathrm{R}=0$ and $\mathrm{R}=2.5$. (a) Load displacement curve; and (b) Rib root tensile strength.
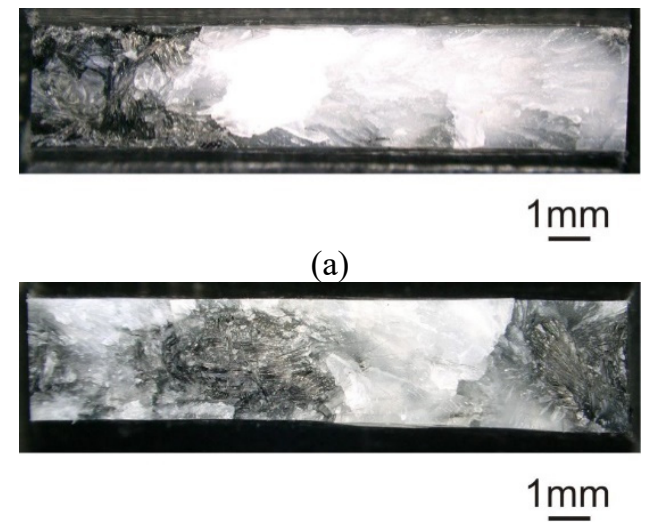

(b)

Figure 8: Fracture surfaces of $\mathrm{T}$-shaped specimens for (a) $\mathrm{R}=0$; and (b) $\mathrm{R}=2.5$. 


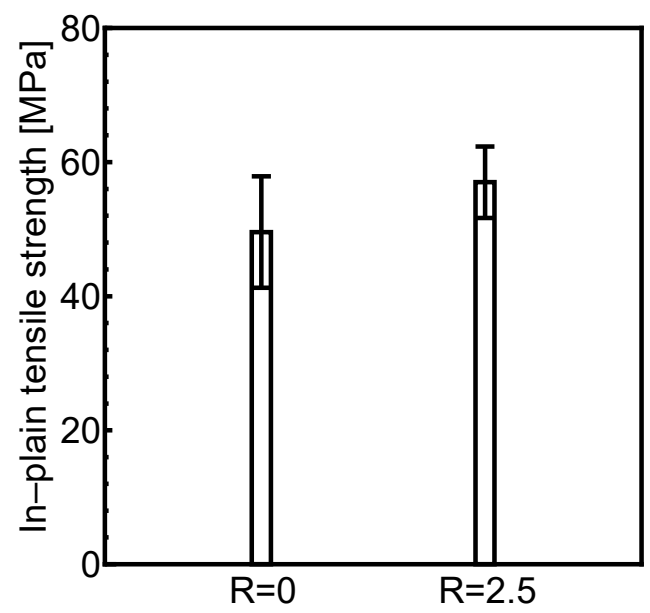

Figure 9: In-plain tensile strength of outer shell laminate.

[\%]

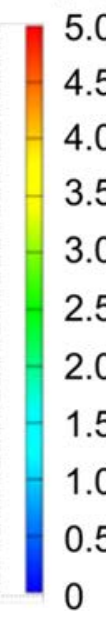

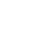

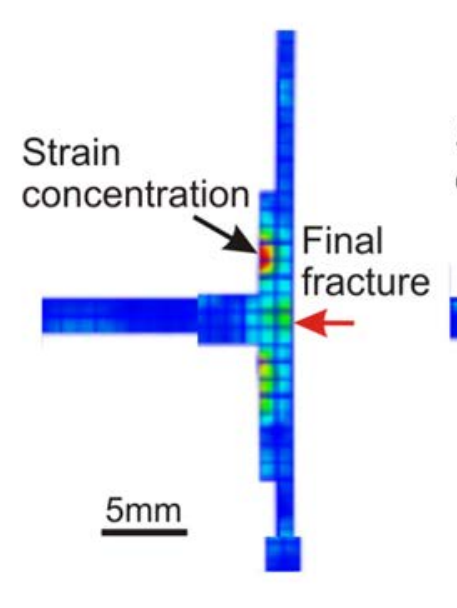

(a)

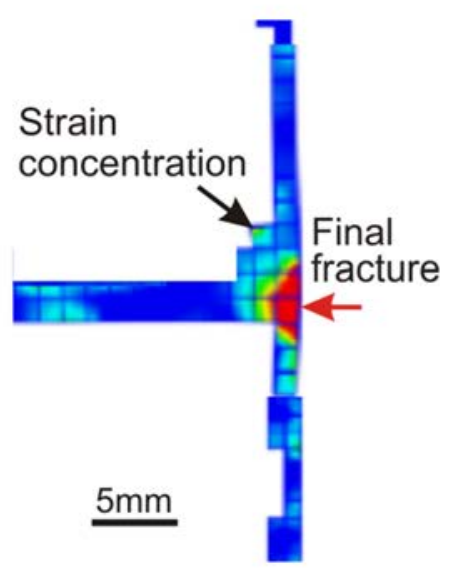

(b)

Figure 10: Strain distribution of the specimens for the in-plain tensile strength of outer shell laminate. (a) $\mathrm{R}=0(\varepsilon=0.3 \%)$; and (b) $\mathrm{R}=2.5(\varepsilon=0.3 \%)$.

The strain concentration at the root of the rib structure of the specimen at $\mathrm{R}=0$ as shown in Fig. 10(b) is larger than that for $\mathrm{R}=2.5$. However, the final fracture occurs from the outer shell laminates at the rib root as shown in Fig. 10 shown with the red arrow.

While the rib root tensile strength of $\mathrm{T}$-shaped specimens for $\mathrm{R}=0$ is higher than that for $\mathrm{R}=2.5$, the in-plain tensile strength of outer shell laminate for $\mathrm{R}=2.5$ is higher than that for $\mathrm{R}=0$. As the effects of rib root shape on the rib root tensile strength and in-plain tensile strength of the outer shell laminate were different, rib root shape should be chosen in accordance to the mold products. 


\section{CONCLUSION}

Carbon fiber reinforced polyamide 6 composites were molded by press and injection hybrid molding by using polyamide 6 random prepreg sheet for outer shell laminate material. Rib root tensile tests of T-shaped specimen and in-plain tensile strength of the outer shell laminate specimens cut out from the molded products were conducted to clarify the effect of rib root shape on their mechanical properties. The investigation yielded the following conclusions:

1. Although the stress concentration at the rib root corner at $\mathrm{R}=0$ is larger than that $\mathrm{R}=2.5$, the $\mathrm{T}$-shaped specimen with the rib root shape for $\mathrm{R}=0$ has the higher rib root tensile strength.

2. While the rib root tensile strength of specimens for $\mathrm{R}=0$ is higher than that for $\mathrm{R}=2.5$, the in-plain tensile strength of outer shell laminate for $\mathrm{R}=2.5$ is higher than that for $\mathrm{R}=0$.

\section{REFERENCES}

[1] Uzawa, K., Present and future state of FRP processing. Journal of the Society of Materials Science, 55(1), pp. 131-137, 2006.

[2] JEC Composites, Composites penetration growth in automotive: towards mass production 2010-2020 trends and forecasts, pp. 13-22, 2011.

[3] International Energy Agency, $\mathrm{CO}_{2}$ Emissions from Fuel Combustion Highlights 2014, pp. 4-6, 2014.

[4] Harte, A.M. \& McNamara, J.F., Over-injection of thermoplastic composites. Journal of Materials Processing Technology, 182(1-3), pp. 12-20, 2007.

[5] Tanaka, K., Fujita, Y. \& Katayama, T., Press and injection hybrid molding of glass fiber reinforced thermoplastics. WIT Transactions on Engineering Sciences, vol. 90, WIT Press: Southampton and Boston, pp. 225-232, 2015.

[6] Tanaka, K., Tokura, D., Katayama, T., Ishikawa, T. \& Tomioka, M., Evaluation of mechanical property of press and injection hybrid molded CF/PA6 using cut prepreg sheets. Journal of the Society of Materials Science, 65(10), pp. 713-720, 2016.

[7] Tanaka, K., Harada, Y., Katayama, T., Ishikawa, T. \& Tomioka, M., Press and injection hybrid molding using CF/PP unidirectional slit prepreg and evaluation of bonding strength at rib root. Journal of the Society of Materials Science, 65(10), pp. 721-726, 2016.

[8] Tomioka, T., Ishikawa, T. \& Kobayashi, T., Fiber-reinforced plastic and production method therefor. World Intellectual Property Organization, publication number $\mathrm{WO} / 2015 / 122500$. 